\title{
CLASSIFYING OPEN PRINCIPAL FIBRATIONS
}

BY

DAVID A. EDWARDS AND HAROLD M. HASTINGS

\begin{abstract}
Let $G$ be a compact metric group. We shall construct classifying spaces for open principal $G$-fibrations over compact metric spaces.
\end{abstract}

1. Introduction. Let $G$ be a compact metric group. J. Milnor [11] gave the first functorial construction of a universal $G$-bundle (classifying space) $E G \rightarrow$ $B G$. R. Milgram [10] gave a later construction with better properties, some of which were developed by N. E. Steenrod [16]. If $G$ is a Lie group, every principal fibration with completely regular total space and with fibre $G$ is a fibre-bundle (A. Gleason [4]) (hence also open), so that $B G$ classifies certain (open) principal $G$-fibrations. We shall extend this result to arbitrary compact metric groups $G$ and compact metric spaces $X$. J. Cohen [1] extensively studied open principal fibrations, and proved, in particular, that every open principal fibration is an inverse limit of fibre-bundles (see \$2). We shall use Cohen's result and some properties of Milgram's resolution (\$4) to construct a universal open principal $G$-fibration

$$
\hat{\xi}_{G}: G \rightarrow \hat{E} G \rightarrow \hat{B} G .
$$

We describe $\hat{\xi}_{G}$ in $\S 2$. In $\S 5-6$ we describe a natural isomorphism

$$
\alpha:[X, \hat{B} G] \stackrel{\approx}{\rightarrow} k_{G}(X),
$$

where $k_{G}(X)$ denotes the class of isomorphism classes of open principal $G$-fibrations over the compact metric space $X$.

(1.3) Remarks. (a) J. P. May [9, see especially \$§4-6] classified principal fibrations up to weak equivalence, a coarser equivalence relation. Compare weak homotopy equivalence versus homotopy equivalence for compact metric spaces.

(b) In $\$ 6$ we shall see that an open principal $G$-fibration over a $\mathrm{CW}$ complex is a fibre-bundle, and hence that there is a natural weak homotopy equivalence $B G \rightarrow \hat{B} G$.

(c) This paper is an outgrowth of the authors' work [3] on strong prohomotopy theory (Steenrod homotopy theory). See also Remark (6.6(b)).

Received by the editors August 11, 1976 and, in revised form, January 13, 1977.

AMS (MOS) subject classifications (1970). Primary 55F15; Secondary 55B05.

Key words and phrases. Lie series, pro-(Lie group), classifying space, Milgram's resolution. 
2. Open principal $G$-fibrations. We recall some of the theory of open principal $G$-fibrations due to J. Cohen [1]. Let $G$ be a compact metric group. Suppose $G$ acts principally (on the right) on a compact metric space $E$; i.e., for any point $y$ in $E$ the orbit $\{y g \mid g \in G\}$ is homeomorphic to $G$. If the quotient map $p: E \rightarrow E / G$ is open and is a Hurewicz fibration (has the covering homotopy property), $p$ is called an open principal G-fibration.

Open principal $G$-fibrations arise naturally as the limits of bundle maps. More precisely, let $\left\{G_{n}\right\}$ be a Lie series for $G(L$. Pontryagin $[13,846])$, i.e., $\left\{G_{n}\right\}$ is a tower of Lie groups bonded by surjections $\pi_{n}$ and $\lim \left\{G_{n}\right\}=G$. (We may use a tower for the Lie series of $G$ because $G$ is a compact metric group.) Assume, without loss of generality, that $G_{0}=\{e\}$.

Cohen associates to an open principal $G$-fibration $G \rightarrow E \rightarrow X$ the tower

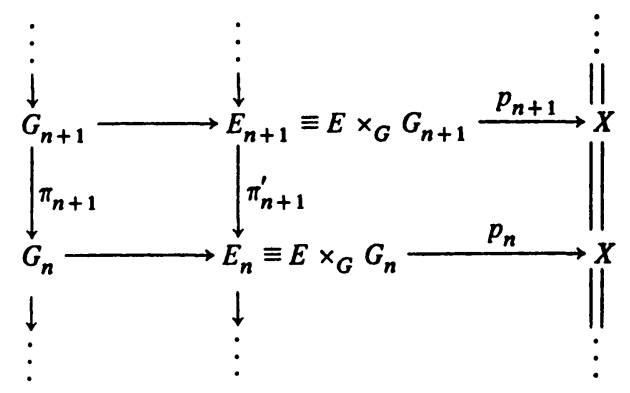

of $G_{n}$-bundles over $X$. We shall frequently call such a tower a principal $G_{n}$-fibration over $X$ and use the notation

$$
\left\{\xi_{n}: G_{n} \rightarrow E_{n} \rightarrow X\right\} \text {. }
$$

(2.3) REMARKS. The maps $\pi_{n+1}$ and $\pi_{n+1}^{\prime}$ above are principal bundle maps with fibre $K_{n+1} \equiv \operatorname{ker}\left(\pi_{n+1}: G_{n+1} \rightarrow G_{n}\right)$.

Conversely, Cohen shows that applying the inverse limit functor lim to the principal $\left\{G_{n}\right\}$-fibration (2.2) yields an open principal $G$-fibration where $G=\lim \left\{G_{n}\right\}$.

We shall call principal $\left\{G_{n}\right\}$-fibrations over a compact metric space $X$ isomorphic if the diagrams (2.1) are level-wise isomorphic over $\mathrm{id}_{x}$. Let $k_{\left\{G_{n}\right\}}(X)$ denote the class of isomorphism classes of principal $\left\{G_{n}\right\}$-fibrations over $X$ and $k_{G}(X)$ the class of isomorphism classes of open principal $G$ fibrations over $X$. Summarizing, we have the following

(2.4) Proposition (J. COHEN [1]). Let $G$ be a compact metric group with Lie series $\left\{G_{n}\right\}$ and let $X$ be a compact metric space. Then there are natural isomorphisms

$$
k_{G}(X) \stackrel{\left\{\cdot \times_{G} G_{n}\right\}}{\stackrel{\lim }{\rightleftarrows}} k_{\left\{G_{n}\right\}}(X) .
$$


Therefore, (1.2) will follow if we can show

$$
[X, \hat{B} G] \cong k_{\left\{G_{n}\right\}}(X) \text {. }
$$

We shall need the following notation. If $H$ is a topological group, let

$$
\xi_{H}: H \rightarrow E H \rightarrow B H
$$

be Milgram's resolution (classifying space) [10] applied to $H$. See $\$ 4$.

Let $G$ be a compact metric group with Lie series $\left\{G_{n}\right\}$. Let

$$
\begin{aligned}
& \xi_{\left\{G_{n}\right\}} \equiv\left\{G_{n} \rightarrow E G_{n} \rightarrow B G_{n}\right\}, \\
& \hat{E} G \equiv \lim \left\{E G_{n}\right\}, \hat{B} G \equiv \lim \left\{B G_{n}\right\},
\end{aligned}
$$$$
\hat{\xi}_{G} \equiv G \rightarrow \hat{E} G_{n} \rightarrow \hat{B} G_{n} \text {, and }
$$$$
\hat{\xi}_{\left\{G_{n}\right\}} \equiv\left\{G_{n} \rightarrow E^{\prime} G_{n} \rightarrow \hat{B} G_{n}\right\}
$$

(take pullbacks of $E G_{n}$ over $\hat{B} G$ ). (Note that $\lim \left\{E^{\prime} G\right\}=\hat{E} G$.)

We shall call $\hat{\xi}_{G}$ the standard universal principal open $G$-fibration, see (1.2), (6.4).

There is a natural map $B G \rightarrow \hat{B} G$. In $\S 6$ we shall see that this map is a weak homotopy equivalence.

(2.6) Proposition. For any compact metric space $X$, any levelwise map of principal $\left\{G_{n}\right\}$-fibrations, or any map of open principal $G$-fibrations over $\mathrm{id}_{X}$ is an isomorphism.

Proof. The first result follows easily from the analogous result in bundle theory; see, e.g., [6, p. 42]. The second result then follows by the proof of Proposition (2.4).

3. Compatible local sections. We shall need suitable versions of local sections and associated partitions of unity for principal $\left\{G_{n}\right\}$-fibrations $\left\{G_{n}\right.$ $\left.\rightarrow E_{n} \rightarrow X\right\}$ over compact metric spaces. An easy inductive argument, using local sections and associated partitions of unity for the principal bundle maps $\pi_{n+1}^{\prime} ; E_{n+1} \rightarrow E_{n}$ associated with a principal $\left\{G_{n}\right\}$-fibration (see (2.1)-(2.3)), yields the following.

(3.1) Proposition. Let $\left\{G_{n} \rightarrow E_{n} \rightarrow X\right\}$ be a principal $\left\{G_{n}\right\}$-fibration over a compact metric space $X$. Then, for each $n \geqslant 0$, there is a finite open cover

$$
\left.\mathscr{U}_{n}=\left\{U_{\left(i_{0}, i_{1}, \ldots, i_{n}\right)}\right) i_{k} \in I_{k}\right\}
$$

a family of local sections.

$$
\delta_{n}=\left\{s_{\left(i_{0}, i_{1}, \ldots, i_{n}\right)}: U_{\left(i_{0}, i_{1}, \ldots, i_{n}\right)} \rightarrow E_{n}\right\},
$$

and a partition of unity

$$
\mathcal{H}_{n}=\left\{h_{\left(i_{0}, i_{1}, \ldots, i_{n}\right)}: X \rightarrow[0,1]\right\}
$$

subordinate to $\mathcal{U}_{n}$, which satisfy the following compatibility conditions. 
$\left(\vartheta_{n}\right) U_{\left(i_{0}, i_{1}, \ldots, i_{n}, i_{n+1}\right)} \subset U_{\left(i_{0}, i_{1}, \ldots, i_{n}\right)}$

$\left(S_{n}\right) \pi_{n+1}^{\prime} \circ s_{\left(i_{0}, i_{1}, \ldots, i_{n}, i_{n+1}\right)}=s_{\left(i_{0}, i_{1}, \ldots, i_{n}\right)}: U_{\left(i_{0}, i_{1}, \ldots, i_{n}, i_{n+1}\right)} \rightarrow E_{n}$.

$\left(\mathcal{C}_{n}\right) h_{\left(i_{0}, i_{1}, \ldots, i_{n}\right)}=\sum_{i_{n+1}} h_{\left(i_{0}, i_{1}, \ldots, i_{n}, i_{n+1}\right)}$

4. Milgram's construction. Following N. E. Steenrod [16], G. Segal [17], S. Mac Lane [7], and [5], we describe the main properties of Milgram's resolution needed later. S. Mac Lane [7] and the second-named author [5] described Milgram's resolution $G \rightarrow E G \rightarrow B G$ of a topological group as the geometric realization (J. Milnor [12]) of a simplicial resolution $G \rightarrow \mathcal{E} G \rightarrow$ BG.

(4.1) Standard simplicial resolutions; see, e.g., [7]. Given a topological group $G$, define a simplicial $G$-space (simplicial object over the category of right $G$-spaces) $\left\{\mathcal{E} G_{n}, d_{i}: \mathcal{E} G_{n} \rightarrow \mathcal{E} G_{n-1}, s_{i}: \mathcal{E} G_{n} \rightarrow \mathcal{E} G_{n+1} \mid n>0,0<i<n\right\}$ by

$$
\begin{aligned}
\mathscr{E} G_{n}=G^{n+1} & =\left\{\left(g_{0}, g_{1}, \ldots, g_{n}\right)\right\}, \\
d_{i}\left(g_{0}, g_{1}, \ldots, g_{n}\right) & =\left(g_{0}, g_{1}, \ldots, g_{i} g_{i+1}, \ldots, g_{n}\right), \\
s_{i}\left(g_{0}, g_{1}, \ldots, g_{n}\right) & =\left(g_{0}, g_{1}, \ldots, g_{i}, e, g_{i+1}, \ldots, g_{n}\right) .
\end{aligned}
$$

Regard $G$ as a constant simplicial object, and let $\mathscr{B} G \equiv \mathscr{E} G \times{ }_{G} *$ be the quotient simplicial space. The sequence $G \rightarrow \mathcal{E} G \rightarrow \mathscr{B} G$ forms the standard simplicial resolution of $G$.

(4.2) Milgram's resolution. Following G. Segal [17] and others, let $R$ : simplicial spaces $\rightarrow C G$ (compactly generated spaces) be the extension of $\mathrm{J}$. Milnor's geometric realization functor [12]. Then [16], [17], [7], [5], the sequence

$$
(G \rightarrow E G \rightarrow B G) \equiv(G=R G \rightarrow R \mathcal{E} G \rightarrow R \mathscr{B} G)
$$

is Milgram's resolution (classifying space) for $G$. The following results show the usefulness of Milgram's construction.

(4.3) Proposition (SteEnrod [16]). Let $G$ and $H$ be topological groups. Then $E(G \times H) \cong E G \times E H$ as $(G \times H)$-spaces, hence $B(G \times H) \cong B G \times$ $B H$.

(4.4) Proposition. Let $p: G \rightarrow H$ be a surjection of Lie groups with kernel $K$. Then the induced map $B p: B G \rightarrow B H$ is a bundle map with fibre $B K$.

Proof (outlined). By L. Pontryagin [13, §44], $G$ is locally isomorphic to $K \times H$ as sets under a local multiplication $K \times H \rightarrow G$. By the proof of (4.3) (in terms of simplicial $G$-spaces), $B G$ is locally isomorphic to $B K \times B H$ near the basepoint. But the map $B p$ is homogeneous because the map $E p: E G \rightarrow$ $E H$ is a map of topological groups [16]. The conclusion follows.

We shall need the following formulas for classifying maps and homotopies between classifying maps. Compare, e.g., [6, pp. 54-57].

(4.5) Alternate coordinates for Milgram's resolution. If $G$ is a topological 
group, the map $G^{n+1} \rightarrow G^{n+1}$ given by

$$
\left(g_{0}, g_{1}, \ldots, g_{n}\right) \mapsto\left(g_{0} g_{1} \cdots g_{n}, g_{1} g_{2} \cdots g_{n}, \ldots, g_{n}\right)
$$

yields an isomorphism of $G$-spaces, where $G$ acts on the rightmost factor of the "domain" $G^{n+1}$, and $G$ acts diagonally (on the right) on the "range" $G^{n+1}$. This yields "delete-repeat" coordinates for $E G$ and, hence, $E G$ with these coordinates:

$$
\begin{aligned}
& \mathcal{E} G=G^{n+1}=\left\{\left(g_{0}, g_{1}, \ldots, g_{n}\right)\right\}, \\
& d_{i}\left(g_{0}, g_{1}, \ldots, g_{n}\right)=\left(g_{0}, g_{1}, \ldots, g_{i-1}, g_{i+1}, \ldots, g_{n}\right) \text {, and } \\
& s_{i}\left(g_{0}, g_{1}, \ldots, g_{n}\right)=\left(g_{0}, g_{1}, \ldots, g_{i-1}, g_{i-1}, g_{i}, g_{i}, g_{i+1}, \ldots, g_{n}\right) .
\end{aligned}
$$

Thus [16] each point in $E G$ may be represented uniquely as $\left(\left(g_{0}, g_{1}, \ldots, g_{n}\right)\right.$, $\left.\left(t_{0}, t_{1}, \ldots, t_{n}\right)\right)$ where $g_{i} \neq g_{i+1}$ and $t_{i} \neq 0$ for all $i$.

(4.6) Classifying maps. As in [6, pp. 54-57], given a principal G-bundle $\xi: G \rightarrow E \rightarrow X$ over a compact metric space $X$, a finite open cover $\mathcal{Q}=\left\{U_{i}\right\}$ of $X$, local sections $s_{i}: U_{i} \rightarrow E$, and a partition of unity $\left\{h_{i}\right\}$ subordinate to थ, there is a canonical classifying map $X \rightarrow B G$ for $\xi$ given by an explicit formula. We omit the details.

(4.7) Linear homotopies. Compare [6, pp. 54-57]. The maps $f_{m, n}\left(G^{m+1} \times\right.$ $\left.\Delta^{m}\right) *\left(G^{n+1} \times \Delta^{n}\right) \rightarrow E G$ defined by

$$
\begin{aligned}
& f_{m, n}\left(\left(\left(g_{0}, g_{1}, \ldots, g_{m}\right),\left(t_{0}, t_{1}, \ldots, t_{m}\right)\right), s,\right.\left.\left(g_{0}^{\prime}, g_{1}^{\prime}, \ldots, g_{n}^{\prime}\right),\left(t_{0}^{\prime}, t_{1}^{\prime}, \ldots, t_{m}^{\prime}\right)\right) \\
&=\left(\left(g_{0}, g_{1}, \ldots, g_{m}, g_{0}^{\prime}, g_{1}^{\prime}, \ldots, g_{m}^{\prime}\right),\right. \\
&\left.\quad\left((1-s)\left(t_{0}, t_{1}, \ldots, t_{m}\right), s\left(t_{0}^{\prime}, t_{1}^{\prime}, \ldots, t_{n}^{\prime}\right)\right)\right)
\end{aligned}
$$

define an equivariant map $\mu: E G * E G$ (diagonal action on the join) $\rightarrow E G$ whose restriction to each factor is the identity.

Now suppose that $f, f^{\prime}: X \rightrightarrows B G$ classify $G \rightarrow E \rightarrow X$. Let $\tilde{f}, \tilde{f^{\prime}}: E \rightrightarrows E G$ be the associated maps on total spaces. Define an equivariant homotopy $H: E$ $\times I \rightarrow E G$ by the formula $H(y, s)=\mu\left(f(y), s, f^{\prime}(y)\right)$. Then the induced map $H: X \times I \rightarrow B G$ provides a canonical "linear homotopy" from $f$ to $f$ ".

5. The transformation $\alpha$. Let $f: X \rightarrow \hat{B} G$ be a continuous map. We may associate to $f$ the principal $\left\{G_{n}\right\}$-fibration $f^{*} \xi_{\left\{G_{n}\right\}}$ over $X$ where $\xi_{\left\{G_{n}\right\}}:\left\{G_{n}\right\} \rightarrow$ $\left\{E^{\prime} G_{n}\right\} \rightarrow \hat{B} G$ is the universal $\left\{G_{n}\right\}$-fibration over $B G$; see $\$ 2$. This yields a function from the set of continuous maps $X \rightarrow \hat{B} G$ to $k_{\left\{G_{n}\right\}}(X)$. In this section we shall prove that homotopic maps $X \rightarrow \hat{B} G$ induce isomorphism principal $\left\{G_{n}\right\}$-fibrations over $X$, and thus define a natural transformation of functors

$$
\alpha:[, \hat{B} G] \rightarrow k_{\left\{G_{n}\right\}}(), \alpha_{X}:[c, \hat{B} G] \rightarrow k_{\left\{G_{n}\right\}}(X)
$$

from compact metric spaces to pointed sets.

(5.1) Proposition. Let $X$ be a compact metric space and let $\xi=\left\{\xi_{n}: G_{n} \rightarrow\right.$ 
$\left.E_{x} \rightarrow X \times I\right\}$ be a principal $\left\{G_{n}\right\}$-fibration over $X \times I$. Let $\xi^{0}=\left\{\xi_{n}^{0}\right\}$ and $\xi^{1}=\left\{\xi_{n}^{1}\right\}$ be the restrictions of $\xi$ to $X \times 0$ and $X \times 1$, respectively. Then there is an isomorphism $\xi^{0} \rightarrow \xi^{1}$.

Proof (outlined). Use Proposition (3.1) and the usual proof that the restrictions of an ordinary bundle over $X \times I$ to $X \times 0$ and $X \times 1$ are isomorphic (e.g., Husemoller [6, pp. 54-57]) to inductively define compatible bundle maps $f_{n}: \xi_{n} \rightarrow \xi_{n}^{1} \subset \xi_{n}$. The required isomorphism is given by $\left\{f_{n} \mid: \xi_{n}^{0}\right.$ $\left.\rightarrow \xi_{n}^{1}\right\}$.

(5.2) Corollary. Let $f, g: X \rightrightarrows \hat{B} G$ be homotopic maps. Then $f$ and $g$ induce isomorphism principal $\left\{G_{n}\right\}$-fibrations over $X$.

Proof. Let $H: X \times I \rightarrow \hat{B} G$ be a homotopy from $f$ to $g$. Then $f^{*} \xi=$ $\left(H^{*} \xi\right)^{0} \cong\left(H^{*} \xi\right)^{1}($ by $(5.1))=g^{*} \xi$.

This yields a well-defined natural transformation $\alpha:[, \hat{B} G] \rightarrow k_{\left\{G_{n}\right\}}()$.

6. Proof of (1.2). We shall prove our main result, that the natural transformation $\alpha:[X, \hat{B} G] \rightarrow k_{\left\{G_{n}\right\}}(X)$ is an isomorphism for compact metric $X$, by constructing classifying maps for principal $\left\{G_{n}\right\}$-fibrations.

(6.1) Construction of classifying maps. Let $\xi=\left\{\xi_{n}: G_{n} \rightarrow E_{n} \rightarrow X\right\}$ be a principal $\left\{G_{n}\right\}$-fibration over a compact metric space $X$.

Use Proposition (3.1) to obtain compatible finite open covers $\mathscr{U}_{n}$, families of local sections $\mathcal{S}_{n}$, and associated partitions of unity $\mathcal{H}_{n}$. Use (4.6) to obtain classifying maps $f_{n}: X \rightarrow B G_{n}$ for each bundle $\xi_{n}: G_{n} \rightarrow E_{n} \rightarrow X$ from this data. A lengthy but straightforward computation shows that the diagrams

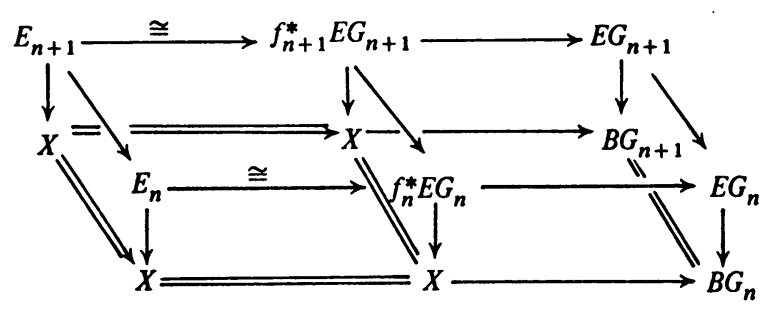

commute. Our construction thus yields the following.

(6.2) Proposition. Let $\xi=\left\{\xi_{n}: G_{n} \rightarrow E_{n} \rightarrow X\right\}$ be a principal $\left\{G_{n}\right\}$-fibration over a compact metric space $X$. Then there is a classifying map $f: X \rightarrow \hat{B} G$ with $\xi \cong f^{*} \xi_{\left\{G_{n}\right\}}$.

Proof. Let $f=\lim _{n}\left\{f_{n}\right\}: X \rightarrow \hat{B} G \equiv \lim _{n}\left\{B G_{n}\right\}$. The conclusion follows.

(6.3) Proposition. Isomorphic principal $\left\{G_{n}\right\}$-fibrations over a compact metric space have homotopic classifying maps. 
Proof. Let $\xi=\left\{\xi_{n}: G_{n} \rightarrow E_{n} \rightarrow X\right\}$ and $\xi^{\prime}=\left\{\xi_{n}^{\prime}: G_{n} \rightarrow E_{n}^{\prime} \rightarrow X\right\}$ be isomorphic principal $\left\{G_{n}\right\}$-fibrations over $X$ "classified" by $f$ and mapping $X$ to $\hat{B} G$, and $\xi \cong f^{*} \hat{\xi}_{G}$, and $\xi^{\prime} \cong f^{*} \hat{\xi}_{G}$. Because $\xi \cong \xi^{\prime}, f^{\prime}\left(=f^{\prime} \circ \operatorname{id}_{X}\right)$ also classifies $\xi$. We obtain a commutative diagram

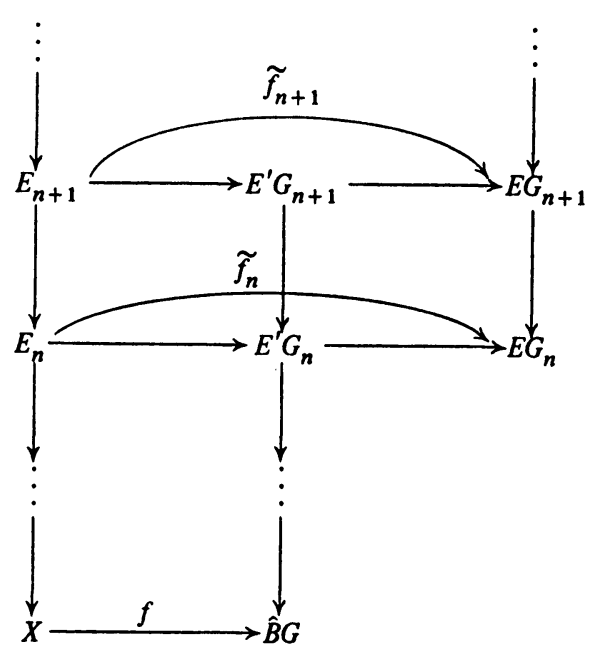

in which $\left\{\tilde{f}_{n}: E_{n} \rightarrow E G_{n}\right\}$ is a compatible family of equivariant maps and a similar diagram involving $f^{\prime}: X \rightarrow \hat{B} G$ and $\left\{\dot{f}_{n}^{\prime}: E_{n} \rightarrow E G_{n}\right\}$. Now use Proposition (4.8) to obtain compatible, equivariant "linear" homotopies $\left\{\tilde{H}_{n}: E_{n} \times\right.$ $\left.I \rightarrow E G_{n}\right\}$ with $\left.H_{n}\right|_{0}=f_{n}$ and $\left.H_{n}\right|_{1}=f_{n}^{\prime}$. Passing to quotient spaces yields compatible homotopies $\left\{H_{n}: X \times I \rightarrow B G_{n}\right\}$. Finally, the required homotopy is given by $H=\lim \left\{H_{n}\right\}: X \times I \rightarrow \hat{B} G\left(\left.H\right|_{0}=f\right.$ and $\left.\left.H\right|_{1}=f^{\prime}\right)$.

(6.4) Proof of (1.2). Propositions (6.2)-(6.3) yield a well-defined function $\beta: k_{G}(X) \rightarrow[X, \hat{B} G]$, where $\beta$ associates to a bundle $\xi$ its classifying map $\beta(\xi) \in[X, \hat{B} G]$. By construction, $\xi \cong \alpha(\beta(\xi)) \equiv \beta(\xi)^{*} \hat{\xi}_{G}$. Also, if $[f] \in$ $[X, \hat{B} G], \beta(\alpha(f))$ and $f$ both classify $f^{*} \hat{\xi}_{G}$, so $[\beta(\alpha(f))]=[f]$ by (6.7). Therefore $\alpha:[X, \hat{B} G] \rightarrow k_{G}(X)$ and $\beta$ are inverse isomorphisms.

(6.5) Proposition. (a) Every open principal G-fibration over a CW complex is a principal G-bundle. (b) The natural map $B G \rightarrow \hat{B} G$ is a weak homotopy equivalence.

Proof. By (1.2) or a direct argument similar to (5.1), $k_{G}$ is a homotopy functor, so each open principal $G$-fibration over a contractible space is a principal $G$-bundle. (a) now follows. (b) now follows from studying open principal $G$-fibrations over spheres.

(6.6) REMARKS. (a) The above classification results can be extended to open principal $G$-fibrations over paracompact spaces.

(b) The classifying space $\hat{B} G=\lim \left\{B G_{n}\right\}$ can be interpreted as the 
homotopy inverse limit [6, §4], holim $\left\{B G_{n}\right\}$, of the tower of fibrations $\left\{B G_{n}\right\}$; see (4.6). In this sense $\hat{B} G$ is a kind of completion of $B G$; consider, for example, profinite groups $G$.

\section{REFERENCES}

1. J. M. Cohen, Inverse limits of principal fibrations, Proc. London Math. Soc. (3) 27 (1973), 178-192. MR 48 \# 7266.

2. A. Dold and R. Lashof, Principal quasi-fibrations and fibre homotopy equivalence of bundles, Illinois J. Math. 3 (1959), 285-305. MR 21 \#331.

3. D. A. Edwards and H. M. Hastings, Cech and Steenrod homotopy theories with applications to geometric topology, Lecture Notes in Math., vol. 542, Springer-Verlag, Berlin and New York, 1976.

4. A. Gleason, Spaces with a compact Lie group of transformations, Proc. Amer. Math. Soc. 1 (1950), 35-43. MR 11, 497.

5. H. M. Hastings, Simplicial topological resolutions, Thesis, Princeton Univ., Princeton, N.J., 1972.

6. D. Husemoller, Fibre bundles, McGraw-Hill, New York, 1966. MR 37 \#4821.

7. S. Mac Lane, The Milgram bar construction as a tensor product of functors, The Steenrod Algebra and Its Applications, Lecture Notes in Math., vol. 168, Springer, Berlin, 1970. MR 42 \#8495.

8. J. P. May, Simplicial objects in algebraic topology, Van Nostrand, Princeton, N.J., 1967. MR 36 \#5942.

9. Classifying spaces and fibrations, Mem. Amer. Math. Soc. 1 (1975), no. 155. MR 51 \#6806.

10. R. J. Milgram, The bar construction and abelian H-spaces, Illinois J. Math. 11 (1967), 242-250. MR 34 \#8404.

11. J. Milnor, Construction of universal bundles. II, Ann. of Math. (2) 63 (1956), 430-436. MR $17,1120$.

12. The geometric realization of a semi-simplicial complex, Ann. of Math. (2) 65 (1957), 357-362. MR 18, 815.

13. L. S. Pontryagin, Topological groups, 2nd ed., Gordon and Breach, New York, 1966. MR 34 \#1439.

14. N. E. Steenrod, The topology of fibre bundles, Princeton Univ. Press, Princeton, N.J., 1951. MR 12, 522.

15. , A convenient category of topological spaces, Michigan Math. J. 14 (1967), 133-152. MR 35 \#970.

16. ___ Milgram's classifying space of a topological group, Topology 7 (1968), 349-368. MR 38 \#1675.

17. G. Segal, Classifying spaces and spectral sequences, Inst. Hautes Études Sci. Publ. Math. No. 34 (1968), 105-112. MR 38 \# 718.

Department of Mathematics, SUNY, Binghamton, New York 13901

Department of Mathematics, Hofstra University, Hempstead, New York 11550 (Current address of H. M. Hastings)

Current address (D. A. Edwards):Department of Mathematics, University of Georgia, Athens, Georgia 30602 